INPLASY

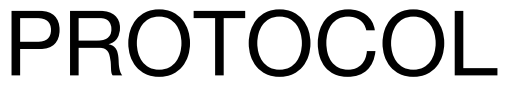

To cite: Wang et al. The efficacy of Tuina for asthma: a protocol for a systematic review and meta-analysis. Inplasy protocol 2020110100. doi:

10.37766/inplasy2020.11.0100

Received: 23 November 2020

Published: 23 November 2020

Corresponding author:

Changhong Wang

741679593@qq.com

Author Affiliation:

Chengdu University of TCM

Support: National Natural

Science Found.

Review Stage at time of this submission: The review has not yet started.

Conflicts of interest:

The authors declare no conflicts of interest.

\section{The efficacy of Tuina for asthma: a protocol for a systematic review and} meta-analysis

Wang, C1; Fan, Z2; Zhao, M³; Jiang, Y4; Wang, Z5; Cheng, Z6; Jiang, $Y^{7}$.

Review question / Objective: Is Tuina effective in improving allergic asthma? which kind of Tuina has the most beneficial effects in allergic asthma?

Condition being studied: Tuina has a long history in China, which has both the function of health and cure, especially in asthma. Consequently, there are a huge number of studies reports the effectiveness of Tuina for allergic asthma, but strongly conclusions have not been achieved owing to a wide variation in scopes, qualities, and outcome.

Information sources: The following database will be searched: PubMed, Web of Science, Embase Cochrane Library, China Biology Medicine disc, China National Knowledge Infrastructure (CNKI), Wanfang, and VIP Data Knowledge Service Platform. Clinical trial registries are part of other resource searches, like Clinical Trials, gov will be searched for ongoing trials with unpublished data.

INPLASY registration number: This protocol was registered with the International Platform of Registered Systematic Review and Meta-Analysis Protocols (INPLASY) on 23 November 2020 and was last updated on 23 November 2020 (registration number INPLASY2020110100).

\section{INTRODUCTION}

Review question / Objective: Is Tuina effective in improving allergic asthma? which kind of Tuina has the most beneficial effects in allergic asthma?
Condition being studied: Tuina has a long history in China, which has both the function of health and cure, especially in asthma. Consequently, there are a huge number of studies reports the effectiveness of Tuina for allergic asthma, but strongly conclusions have not been achieved owing 
to a wide variation in scopes, qualities, and outcome.

\section{METHODS}

Participant or population: This review includes allergic asthma patients, no matter what reason caused allergic asthma.

Intervention: Tuina-related was the main intervention. the duration and frequency of therapy are not limited.

Comparator: There is no exclusion based on comparator method for this review, and the patients could be treated with any type of control group including Tuina.

Study designs to be included: Randomized controlled trials with no limitations on blinding or publication types will be included.

Eligibility criteria: Randomized controlled trials (RCTs) with no limitations on binding published in English and Chinese up to November 20, 2020 will be included. Patients with primary diseases related allergic asthma will be include, regardless of the underlying diseases, age, gender, education, ethnicity, and occupation. There will be no restrictions regarding language or publication status. Non-randomized trials, case reports, observational studies, and reviews will be excluded.

Information sources: The following database will be searched: PubMed, Web of Science, Embase Cochrane Library, China Biology Medicine disc, China National Knowledge Infrastructure (CNKI), Wanfang, and VIP Data Knowledge Service Platform. Clinical trial registries are part of other resource searches, like Clinical Trials, gov will be searched for ongoing trials with unpublished data.

Main outcome(s): The main criteria are: (1) Symptoms and signs disappear; (2) No significant wheezing after the event; (3) Stop the drug for 3 months without attack; (4) Film degree exam; (5) Lung ventilation.
Additional outcome(s): Secondary assessment criteria include Lung sounds and cough disappear. At the same time, close attention should be paid to whether adverse reactions or adverse events occur during the experiment to comprehensively evaluate the clinical efficacy and safety of acupuncture in the treatment of allergic asthma.

Data management: According to the guidance of the Cochrane Handbook for Systematic Review of Interventions, the quality of included RCTs will be assessed by 2 reviewers (YG and LC). The items of quality including inclusion criteria, sample size estimation, baseline, randomization, allocation sequence concealment, blinding, selective reporting, missing data management, and other bias. Evidence quality will be shown as high, unclear, low risk of bias in accordance with the criteria of the risk judgment. If there are disagreements, an experienced researcher (YJ) will make the decision.

Strategy of data synthesis: The Revman $\mathbf{5 . 3}$ software provided by Cochrane Collaboration is used to perform all statistical analyses. All outcomes are presented as continuous variables in this review. We will perform a pairwise metaanalysis using a random-effect model. To determine the effect size, risk rations with $95 \%$ confidence intervals will be calculated for dichotomous outcomes and the standard mean difference with $95 \%$ confidence intervals will be calculated for continuous outcomes. Depending on the heterogeneity assessed by the 12 statistic, a fixed- ore random- effect model will be used. If there is statistical heterogeneity, sensitivity to explore the source of heterogeneity.

Subgroup analysis: If the heterogeneity is apparent, subgroup and meta-regression analysis will be set up according to the characteristic of the study to explore the source of heterogeneity with regard to age, gender, region, type of control interventions, type of Tuina, and frequency and duration. 
Sensibility analysis: For the quality analysis, we will conduct a sensitivity analysis of main outcomes to explore an individual study's influence of bias on results.

Language: English and Chinese.

Country(ies) involved: China.

Keywords: allergic asthma, Tuina, protocol, systematic review, meta-analysis.

Contributions of each author:

Author 1 - Changhong Wang - The author drafted the manuscript.

Author 2 - Zhipeng Fan - The author provided statistical expertise.

Author 3 - Mao Zhao - The author contributed to the development of the selection criteria, and the risk of bias assessment strategy.

Author 4 - Yuchang Jiang - The author provided statistical expertise.

Author 5 - Zhaodi Wang - The author provided statistical expertise.

Author 6 - Zhaoxing Cheng - The author contributed to the development of the selection criteria, and the risk of bias assessment strategy.

Author 7 - Yong Jiang - The author read, provided feedback and approved the final manuscript. 